\title{
An Analysis of Demographics and Inhaled Corticosteroid Use on COVID-19 Outcomes Among Hospitalized Adult Asthmatics: An Early Experience at a NY Hospital System
}

\author{
Sherry Farzan (1D ${ }^{1-4}$ \\ André Rebaza ${ }^{2,5}$ \\ Shipra Rai ${ }^{2}$ \\ Maria Santiago ${ }^{2,5}$ \\ Northwell Health COVID-I9 \\ Research Consortium \\ 'Division of Allergy \& Immunology, \\ Donald and Barbara Zucker School of \\ Medicine at Hofstra/Northwell, Great \\ Neck, NY, USA; ${ }^{2}$ Department of \\ Pediatrics, Donald and Barbara Zucker \\ School of Medicine at Hofstra/Northwell, \\ New Hyde Park, NY, USA; ${ }^{3}$ Department \\ of Medicine, Donald and Barbara Zucker \\ School of Medicine at Hofstra/Northwell, \\ Manhasset, NY, USA; ${ }^{4}$ Institute of Health \\ System Science, Feinstein Institutes for \\ Medical Research/Northwell Health, \\ Manhasset, NY, USA; ${ }^{5}$ Division of Pediatric \\ Pulmonology, Donald and Barbara Zucker \\ School of Medicine at Hofstra/Northwell, \\ New Hyde Park, NY, USA
}

Correspondence: Sherry Farzan Department of Pediatrics, Division of Allergy \& Immunology, Donald and Barbara Zucker School of Medicine at Hofstra/Northwell, 865 Northern Blvd. Suite 101, Great Neck, NY, II02I, USA

Tel + I 516-622-5070

Fax+I 516-622-5060

Email sfarzan@northwell.edu
Purpose: It is unclear whether asthma is a risk factor for the development of COVID-19; however, severe asthma is a risk factor for morbidity and mortality. While systemic corticosteroids are beneficial during the inflammatory phase of COVID-19, the impact of inhaled corticosteroids (ICS) is unclear. We sought to characterize asthmatics admitted with COVID19 early in the pandemic, determine if baseline factors are associated with more severe COVID-19 disease, and if the use of ICS may mitigate the severity of COVID-19.

Patients and Methods: A retrospective chart review of hospitalized asthma patients $>18$ years testing positive for SARS-CoV2 from March to June 2020 was performed. Baseline demographic and asthma variables were collected. COVID-19 outcomes and laboratory values were extracted and compared between sex, race, ethnicity, and ICS use.

Results: Of the 906 patient charts reviewed, 787 asthmatics were confirmed to be admitted for symptomatic COVID-19. Sex differences were found in hospitalization and intubation. Non-Hispanic patients had a significantly greater number of days on ventilator. Patients on ICS were 1.6 times more likely to be discharged on supplemental oxygen compared to patients not on ICS ( $p=0.01)$.

Conclusion: While our findings confirm trends observed by others with respect to risk factors among asthmatics with COVID-19, differences based on sex, ethnicity and ICS use in asthmatics were observed. Our finding that ICS use was associated with discharge with oxygen is novel. Future research is needed to study the trajectory of asthmatics from diagnosis to outcomes.

Keywords: asthma, COVID-19, sex, inhaled corticosteroids, outcomes

\section{Introduction}

Over the past 18 months, the COVID-19 pandemic has had significant repercussions, with over 170 million cases and over 3.5 million deaths. Given the respiratory transmission of SARS-CoV2 and the role of respiratory viruses as triggers for asthma exacerbations, there is legitimate concern that COVID-19 would result in worse outcomes among asthmatics. The Centers for Disease Control has designated moderate-to-severe asthma as a factor that increases the risk of developing severe COVID-19. ${ }^{1}$

Data regarding COVID-19 in asthmatics are mixed, and conclusions about the effects of the SARS-CoV2 virus among this population are difficult to make. Early 


\section{Graphical Abstract}

\section{An Analysis of Demographics and Inhaled Corticosteroid (ICS) Use on COVID-19 Outcomes Among Asthmatics}

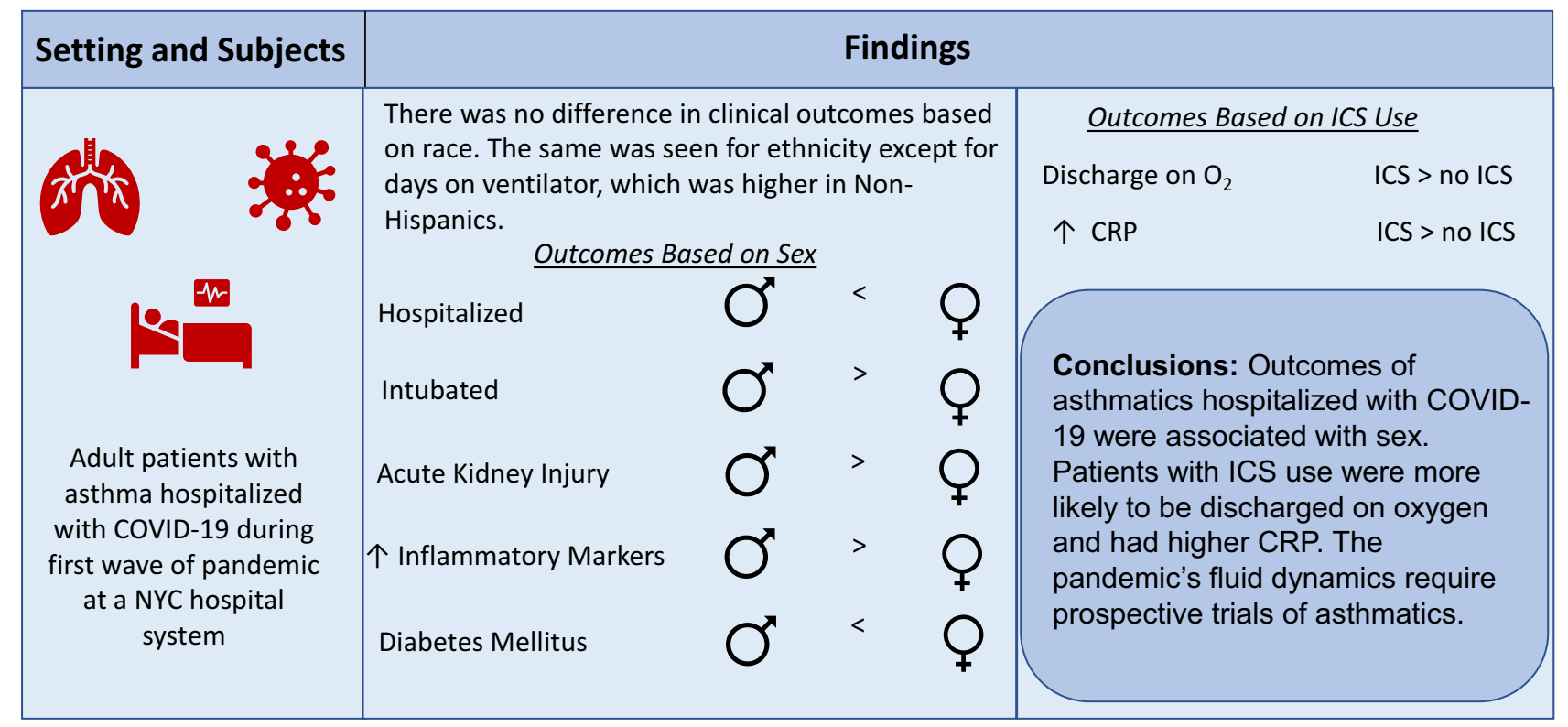

reports from Wuhan demonstrated a lower prevalence of asthmatics among those diagnosed with COVID-19 compared to the general prevalence. ${ }^{2}$ While an early cohort of COVID-19 patients in our institution demonstrated a prevalence of asthma similar to that of the general population, ${ }^{3}$ a study from the United Kingdom (UK) reported a higher prevalence. ${ }^{4}$ A systematic review of international studies showed a prevalence of $1.6 \%$. $^{5}$ The wide range is likely due to demographic differences and variability in the timing of the various studies. ${ }^{2-5}$

Multiple studies have also addressed the impact of a history of asthma on the severity of COVID-19 disease. Early retrospective studies did not demonstrate that a history of asthma was associated with more severe disease, intensive care unit (ICU) admission, mechanical ventilation or increased mortality. ${ }^{6-10}$ However, several studies have identified severe asthma (defined as oral corticosteroid use in the past year, or GINA 4/5) as a risk factor for COVID-19 hospital death, ICU admission or mechanical ventilation. ${ }^{11-}$ 13 Another study noted asthma was a risk factor for hospitalization among elderly women. ${ }^{14}$

Corticosteroids, both inhaled and systemic, play a critical role in the management of asthma control and exacerbations, respectively. Given its role as an immunosuppressant, a valid question is whether pre-existing corticosteroid use can amplify or mitigate COVID-19 disease. The RECOVERY trial and others have clearly demonstrated that dexamethasone significantly reduces the risk of death during the inflammatory phase of COVID-19. ${ }^{15,16}$ However, there is a dearth of clinical data regarding the modifying role of inhaled corticosteroids (ICS) on COVID-19 outcomes. In a nested case-control study, the use of ICS among asthma and COPD patients was not associated with greater risk of COVID-19 death. ${ }^{17}$ Ciclesonide, an ICS, blocks in vitro viral replication of SARS-CoV2 in a concentration-dependent manner, but other corticosteroids, fluticasone and dexamethasone, do not. $^{18,19}$ SARS-CoV2 infection of respiratory epithelial cells is dependent on angiotensin-converting enzyme 2 (ACE-2) and transmembrane serine protease 2 (TMPRSS2) proteins, for attachment and priming of the $\mathrm{S}$ protein, respectively. ${ }^{20}$ ACE-2 is downregulated in atopic asthma and allergic rhinitis ${ }^{21}$ and may be mediated by the Type 2 cytokine, interleukin $13 .{ }^{22}$ Use of ICS was associated with decreased gene expression of ACE-2 and TMPRSS2 in a dose-dependent manner among asthmatics. ${ }^{23}$ Based on this preclinical data and limited clinical data, the role of 
ICS in asthmatics with COVID-19, as well as nonasthmatics, deserves further attention.

Given the conflicting data regarding asthma as a risk factor for severe COVID-19 disease and the questions surrounding the role of inhaled corticosteroids in this population, the current study aims to describe the demographic and clinical picture of asthmatics admitted to one New York hospital system with COVID-19 early in the pandemic. We hypothesize that certain demographic and clinical factors are associated with more severe COVID-19 disease and that the use of ICS may mitigate the severity of COVID-19.

\section{Materials and Methods}

A retrospective cohort chart review was conducted of patients $>18$ years of age with a diagnosis of asthma who tested positive for SARS-CoV2 by PCR and were admitted to 1 of 12 Northwell Health System hospitals for symptomatic COVID-19 between March and June 2020. Using de-identified data, electronic medical record (EMR) review was performed by 15 physician reviewers. Asthmatic patients were defined as patients $>18$ years of age with a diagnosis of asthma documented in the past medical history section of the EMR. Data was entered and managed by the Research Electronic Data Capture (REDCap) at Northwell Health. Baseline variables included patient's age, gender, race, ethnicity, body mass index (BMI), co-morbidities, and smoking status. Asthma-related variables included controller medication and total ICS dose expressed as beclomethasone equivalents $(\mu \mathrm{g} / \mathrm{kg} / \mathrm{day})$. COVID-19-related variable endpoints included length of illness before presentation, length of stay (LOS), ICU admission, days in ICU, need for mechanical ventilation, days on a ventilator, medical management for COVID-19 (eg, hydroxychloroquine, azithromycin, remdesivir), radiographic findings on chest $\mathrm{x}$-ray (CXR), and discharge disposition, including mortality. Laboratory markers evaluated included initial and peak measurements of white blood cell count (WBC), initial and nadir absolute lymphocyte count (ALC), absolute neutrophil count (ANC), C-reactive protein (CRP), erythrocyte sedimentation rate (ESR), aspartate aminotransferase (AST), alanine aminotransferase (ALT), ferritin, D-dimer, procalcitonin, troponin, as well as cytokine panels and $\mathrm{T}$ and $\mathrm{B}$ subsets. Outcomes and laboratory variables were compared between gender, race, ethnicity, and ICS controller use. We also evaluated differences in baseline demographic and COVID-19-related endpoints based on daily ICS dose. The study was approved by the
Northwell Institutional Review Board under the umbrella of the Northwell COVID-19 Research consortium. As per the Declaration of Helsinki, which introduced the idea of independent ethical committee review of research studies, this project was reviewed by the Northwell Health IRB (FWA \#00002505) and deemed exempt as per 45 CFR 46.104(d)(4) (III). All identifiable health information was kept confidential.

\section{Statistical Analysis}

Summary statistics were reported for demographics, laboratory variables, CXR results, and COVID-19-related endpoints. Continuous variables were reported as means and standard deviations. Categorical variables were reported as frequencies and percentages. For continuous variables, $t$-tests were used to test for differences in distribution between male and female asthmatics. For categorical variables, Chi-square tests or Fisher's exact tests were used, as appropriate, to compare outcomes and laboratory values. Logistic regression analysis was used to determine correlations between daily beclomethasone equivalent dose and outcomes, as well as initial laboratory values and outcomes. A p-value $<0.05$ was considered statistically significant. All analyses were conducted using $\mathrm{R}$ version 1.4.1717.

\section{Results \\ Subject Identification}

Nine hundred and six patients were identified as adult asthmatics admitted to the Northwell Health hospitals with a positive nasopharyngeal PCR for SARS-CoV2 between March 2020 and June 2020. Of the 906 patient charts reviewed, 820 patients were confirmed as asthmatic. Of these, 787 patients were confirmed to be admitted for symptomatic COVID-19. Thirty-three charts were excluded due to admission for non-COVID-19 reasons.

\section{Baseline Characteristics}

Of the 787 asthmatics admitted for symptomatic COVID-19, $39.6 \%$ were male. The mean age of asthmatics was 59.8 years, with females being older than males (mean 61 versus 58 years, $\mathrm{p}=0.01$, Table 1 ). A significantly higher proportion of Black patients were female and a significantly higher proportion of White patients were male $(p=0.01)$. There were more active and former male smokers than female smokers $(p=0.04)$. There was no difference between sexes with respect to ethnicity, BMI, or COVID-19 exposure. 
Table I Demographics, Treatment and Clinical Outcomes in Adult Asthmatics Admitted with COVID-19

\begin{tabular}{|c|c|c|c|c|}
\hline & $\begin{array}{c}\text { Overall } \\
n=787\end{array}$ & $\begin{array}{c}\text { Male } \\
n=3 \mid 2\end{array}$ & $\begin{array}{c}\text { Female } \\
n=475\end{array}$ & $\mathrm{p}$ value \\
\hline Age (years), Mean (SD) & $59.8(16.4)$ & $58(16.0)$ & $61(16.5)$ & 0.01 \\
\hline Race, n (\%) & & & & $0.01^{\mathrm{a}}$ \\
\hline Asian & $65(8.3)$ & $33(10.6)$ & $32(6.7)$ & \\
\hline Black & $203(25.8)$ & $66(21.2)$ & $137(28.8)$ & \\
\hline White & $264(33.5)$ & $118(37.8)$ & $146(30.7)$ & \\
\hline Other/Unknown* & $255(32.4)$ & $95(30.4)$ & $160(33.7)$ & \\
\hline Ethnicity, n (\%) & & & & 0.52 \\
\hline Hispanic/Latino & $17 \mid(2 \mid .7)$ & $62(19.9)$ & $109(22.9)$ & \\
\hline Non-Hispanic or Latino & $573(72.8)$ & $231(74.0)$ & $342(72.0)$ & \\
\hline Other & $43(5.5)$ & $19(6.1)$ & $24(5.1)$ & \\
\hline BMI, Mean (SD)** & $31.9(9.3)$ & $31.2(7.4)$ & $32.4(10.4)$ & 0.07 \\
\hline Tobacco Status, n (\%) & & & & $0.04^{\mathrm{b}}$ \\
\hline Active & $20(2.5)$ & $12(3.8)$ & $8(1.7)$ & \\
\hline Former & $112(14.2)$ & $52(16.7)$ & $60(12.6)$ & \\
\hline Never/Unknown*** & $655(83.2)$ & $248(79.5)$ & $407(85.7)$ & \\
\hline COVID-19 Exposure, n (\%) & $232(29.5)$ & $82(27.2 \%)$ & $150(32.8)$ & 0.12 \\
\hline IF YES to Exposure, n (\%) & & & & 0.60 \\
\hline Household member & $168(72.4)$ & 57 (69.5\%) & III (74) & \\
\hline Non-Household member & $62(36.9)$ & $24(29)$. & $38(26)$ & \\
\hline Days illness, Mean (SD) & $6.84(5.0)$ & $7.29(5.3)$ & $6.53(4.8)$ & 0.05 \\
\hline \multicolumn{5}{|l|}{ Asthma Controller Medications, $\mathbf{n}(\%)$} \\
\hline Montelukast & $205(26.0)$ & $70(22.4)$ & $135(28.4)$ & 0.07 \\
\hline ICS & $38(4.8)$ & II (3.5) & $27(5.7)$ & 0.23 \\
\hline$I C S+L A B A$ & $263(33.4)$ & $97(3 \mathrm{I} .1)$ & $166(34.9)$ & 0.30 \\
\hline ICS or ICS + LABA & $301(38.2)$ & $108(34.6)$ & $193(40.6)$ & 0.10 \\
\hline Number of Controllers, $n$ (\%) & & & & 0.10 \\
\hline 0 & $422(53.6)$ & I8I (58.0) & $24 I(50.7)$ & \\
\hline I & $179(22.7)$ & $72(23.1)$ & $107(22.5)$ & \\
\hline 2 & $127(16.1)$ & $43(13.7)$ & $84(17.7)$ & \\
\hline 3 & $52(6.6)$ & $14(4.5)$ & $38(8.0)$ & \\
\hline 4 & $7(0.9)$ & $2(0.7)$ & $5(1.1)$ & \\
\hline Number of Controllers, n (\%) & & & & 0.05 \\
\hline 0 & $422(53.6)$ & $175(56.1)$ & $232(48.8)$ & \\
\hline Any & $365(46.4)$ & 137 (43.9) & $243(5 \mid .2)$ & \\
\hline Ventilator Support, n (\%) & $145(18.4)$ & $69(22.1)$ & $76(16.0)$ & 0.04 \\
\hline Days on Ventilator, Mean (SD)**** & $13.9(15.2)$ & $12.3(11.1)$ & $15.4(18.2)$ & 0.20 \\
\hline \multicolumn{5}{|l|}{ Medical Management, n (\%) } \\
\hline Plaquenil & $604(76.7)$ & $246(78.9)$ & $358(75.4)$ & 0.30 \\
\hline Azithromycin & $379(48.2)$ & $147(47.1)$ & $232(48.8)$ & 0.69 \\
\hline Remdesivir & $8(1.0)$ & $4(1.3)$ & $4(0.8)$ & 0.81 \\
\hline Anti IL-I & $78(9.9)$ & $34(10.9)$ & $44(9.3)$ & 0.53 \\
\hline Anti IL-6 & $85(10.8)$ & $45(14.4)$ & $40(8.4)$ & 0.01 \\
\hline Steroids & $339(43.1)$ & $135(43.3)$ & $204(43.0)$ & 0.99 \\
\hline Convalescent Plasma & $17(2.2)$ & $9(2.9)$ & $8(1.7)$ & 0.38 \\
\hline
\end{tabular}

(Continued) 
Table I (Continued).

\begin{tabular}{|c|c|c|c|c|}
\hline & $\begin{array}{c}\text { Overall } \\
n=787\end{array}$ & $\begin{array}{c}\text { Male } \\
n=312\end{array}$ & $\begin{array}{c}\text { Female } \\
n=475\end{array}$ & p value \\
\hline Discharged on Oxygen, $\mathrm{n}(\%)$ & $131(16.6)$ & $46(15.6)$ & $85(18.8)$ & 0.30 \\
\hline Discharged with Tracheostomy, n (\%) & $15(1.9)$ & $7(2.4)$ & $8(1.8)$ & 0.74 \\
\hline Length of Stay (days), Mean (SD) & $9.65(11.0)$ & $9.84(11.4)$ & $9.51(10.8)$ & 0.68 \\
\hline ICU Admission, n (\%) & $194(24.7)$ & $89(28.5)$ & $105(22.1)$ & 0.05 \\
\hline Deaths, n (\%) & I44 (I8.3) & $62(19.9)$ & $82(17.3)$ & 0.39 \\
\hline
\end{tabular}

Notes: ${ }^{a}$ Chi-square analysis performed for race and sex. There were significantly more White males than females and more Black females than males in the cohort. ${ }^{b} \mathrm{Chi}-$ square analysis performed for tobacco status and sex. There were more active and former male smokers than female smokers. *3। Patients with "Unknown" race were grouped together with "Other". **Only 65I patients had BMI recorded in the cohort and thus were included in the analysis. ***50 Patients with "Unknown" smoking status were grouped together with patients with "Never" status. ****t-test was performed on patients who required ventilator support during admission. Patients without ventilator support were excluded from analysis.

Abbreviations: COVID-19, coronavirus disease 2019; SD, standard deviation; BMI, body mass index; ICS, inhaled corticosteroids; ICS + LABA, inhaled corticosteroids plus long-acting beta agonist; ICU, intensive care unit; IL, interleukin.

\section{Asthma Controller Medications}

About half of patients (53.6\%) were not on any controller medication on admission (Table 1). Of those on a daily controller medication, $22.7 \%, 16.1 \%, 6.6 \%$ and $0.9 \%$ were on one, two, three or four controller medications, respectively. Approximately $38 \%$ of the patients were on either ICS or inhaled corticosteroid/long-acting beta agonist (ICS/LABA), but most were on combination therapy.

\section{COVID-19 Outcomes}

Overall, $18.4 \%$ of the patients required ventilator support, with more males requiring intubation than females $(22.1 \%$ versus $16 \%, \mathrm{p}=0.04$, Table 1). Asthmatics received many treatments for COVID-19, including hydroxychloroquine (76.7\%), azithromycin (48.2\%), steroids (43.1\%), anti-IL 6 monoclonal antibody (10.8\%), anti-IL 1 monoclonal antibody (9.9\%), convalescent plasma (2.2\%) and remdesivir (1.1\%). There were no differences between males and females in terms of the medications received for treatment of COVID-19, except for anti-IL 6 monoclonal antibody, which was higher in men compared to women $(14.4 \%$ versus $8.4 \%, \mathrm{p}=0.01)$. Approximately $25 \%$ of the patients were admitted to the ICU. Mean hospital length of stay was 9.65 days, and most patients were discharged home. There were no differences with respect to disposition, supplemental oxygen on discharge, LOS, ICU admission, or in mortality between men and women.

When comparing clinical COVID-19 outcomes based on ethnicity and race, there was no difference in ventilator support, mortality, supplemental oxygen on discharge, tracheostomy on discharge, LOS, or ICU admission (Tables 2 and 3). However, non-Hispanics had significantly greater number of days on ventilator than Hispanics (15 versus 11 days, $p=0.04)$. There was no difference based on race.

\section{Radiographic Findings and Laboratory Results}

Most (73.7\%) asthmatics had an initial CXR demonstrating infiltrates, opacities, or consolidations (Table 4). And, 23.8\%

Table 2 Clinical Variables Based on Ethnicity

\begin{tabular}{|c|c|c|c|}
\hline & $\begin{array}{c}\text { Hispanic } \\
n=|7|\end{array}$ & $\begin{array}{c}\text { Non-Hispanic } \\
n=573\end{array}$ & p value \\
\hline Ventilator Support, n (\%) & $33(19.3)$ & III (19.4) & 1.00 \\
\hline Days on Ventilator, Mean (SD) ${ }^{\mathrm{a}}$ & $10.6(8.5)$ & $14.9(16.8)$ & 0.04 \\
\hline Deaths, n (\%) & $29(17)$ & $109(19)$ & 0.61 \\
\hline Discharged on Oxygen, $\mathrm{n}(\%)$ & $31(18.1)$ & $93(16.2)$ & 0.57 \\
\hline Discharged Tracheostomy, n (\%) & I (0.6) & $13(2.3)$ & 0.21 \\
\hline Length of Stay (days), Mean (SD) & $8.9(9.1)$ & $10.2(11.8)$ & 0.13 \\
\hline ICU Admission, n (\%) & $38(22.2)$ & $150(26.2)$ & 0.35 \\
\hline
\end{tabular}

Note: ${ }^{a}$ Analysis was performed on patients with ventilator support during admission. Patients without ventilator support were excluded.

Abbreviations: SD, standard deviation; ICU, intensive care unit. 
Table 3 Clinical Variables Based on Race

\begin{tabular}{|l|c|c|c|c|c|}
\hline & $\begin{array}{c}\text { Asian } \\
\mathbf{n = 6 5}\end{array}$ & $\begin{array}{c}\text { Black } \\
\mathbf{n = 2 0 3}\end{array}$ & $\begin{array}{c}\text { White } \\
\mathbf{n = 2 6 4}\end{array}$ & $\begin{array}{c}\text { Other } \\
\mathbf{n = 2 2 4}\end{array}$ & p value \\
\hline Ventilator Support, n (\%) & $18(27.7)$ & $35(I 7.2)$ & $47(17.8)$ & $45(20.1)$ & 0.26 \\
Days on Ventilator, Mean (SD) & $15.6(I I .6)$ & $14.7(I 7.2)$ & $14.6(18.9)$ & $11.9(10.1)$ & 0.73 \\
Deaths, n (\%) & $18(27.7)$ & $29(14.3)$ & $53(20.1)$ & $43(19.2)$ & 0.09 \\
Discharged on Oxygen, n (\%) & $8(I 2.3)$ & $37(I 8.2)$ & $4 I(I 5.5)$ & $40(17.9)$ & 0.59 \\
Discharged Tracheostomy, n (\%) & $2(3.1)$ & $5(2.5)$ & $5(I .9)$ & $3(1.3)$ & 0.68 \\
Length of Stay (days), Mean (SD) & $12.2(14.6)$ & $9.87(I I .2)$ & $9.35(10.6)$ & $9.5 I(I 0.7)$ & 0.32 \\
ICU Admission, n (\%) & $24(36.9)$ & $50(24.6)$ & $59(22.3)$ & $57(25.4)$ & 0.12 \\
\hline
\end{tabular}

Notes: ${ }^{b}$ Analysis was performed on patients with ventilator support during admission. Patients without ventilator support were excluded.

Abbreviations: SD, standard deviation; ICU, intensive care unit.

of the patients developed acute kidney injury during their hospitalization, with a significantly higher proportion in males compared to females ( $34 \%$ versus $19.7 \%, \mathrm{p}<0.001)$. Asthmatics developed typical laboratory abnormalities consistent with COVID-19, including elevated ESR, D-dimer, ferritin, lymphopenia, neutrophilia, and liver function test abnormalities (Table 4). Men had a higher initial ALT than women (56.8 U/L versus $40.7 \mathrm{U} / \mathrm{L}, \mathrm{p}<0.001)$, initial ferritin (1480 ng/mL versus $706 \mathrm{ng} / \mathrm{mL}, \mathrm{p}<0.001$ ), peak ferritin (2610 ng/mL versus $1120 \mathrm{ng} / \mathrm{mL}, \mathrm{p}<0.001$ ) and peak $\mathrm{D}$-dimer (5140 versus $3200, \mathrm{p}=0.02$ ). $\mathrm{T}$ cell enumeration was performed in a limited number of patients, but CD4 numbers were observed to be significantly higher in women than men $(432$ cells $/ \mu \mathrm{L}$ versus 273 cells $/ \mu \mathrm{L}, \mathrm{p}=0.004)$.

\section{Comorbidities}

The most common comorbidities among asthmatics were hypertension (56.3\%), diabetes mellitus (32.5\%) and obesity $(27.6 \%)$ (Table 5 ). There was no difference in the prevalence of comorbidities except for diabetes, which was more prevalent in females compared to males $(36 \%$ versus $27.2 \%, \mathrm{p}=0.01$ ).

\section{Inhaled Corticosteroids and Beclomethasone Equivalent Dose}

Patients on ICS were older (63.5 years versus 57.6 years, $\mathrm{p}<0.001$, Table 6). Treatment with ICS did not differ based on race or ethnicity and did not predict COVID-19 outcomes in terms of length of stay, ICU admission, ventilator support, days on ventilator, need for tracheostomy or mortality. However, patients on ICS were 1.6 times more likely to be discharged on supplemental oxygen compared to patients not on ICS, after adjusting for age $(\mathrm{p}=0.01)$. The beclomethasone equivalents of the ICS were not related to COVID-19 endpoints such as ICU admission, ventilator support, oxygen on discharge, tracheostomy, mortality, or chest x-ray findings (Supplemental Table 1).

\section{Initial Lab Markers on COVID-19 Endpoints}

Logistic regression analysis demonstrated ICU admission, need for ventilation, and death were more likely the higher the initial WBC $(p<0.001, p=0.002, p=0.007$, respectively, Supplemental Table 2A-D). Similar findings were seen with initial AST, ferritin, and procalcitonin values. ICU admission was more likely the higher the initial ALT value $(\mathrm{p}=0.02)$. A similar relationship was noted in ICU admission and need for ventilation with initial troponin values $(\mathrm{p}=0.04$ and $\mathrm{p}=0.02$, respectively). Analysis also revealed mortality was more likely as initial D-dimer increased $(\mathrm{p}=0.03)$. Analysis of lab markers and ICS use in the cohort revealed patients with ICS use had higher initial and peak CRP levels compared to patients without ICS use $(p=0.006$ and $\mathrm{p}=0.002$, respectively, Supplemental Table 3).

\section{Discussion}

Asthma is one of the most common chronic respiratory diseases in the United States. During the pandemic, it has been reasonable to assume that a virus transmitted through respiratory particles and affects the respiratory epithelium could have a significant impact on the health of asthmatics. This retrospective study is one of the most comprehensive descriptive studies regarding adult asthmatic inpatients hospitalized for COVID-19, and sheds light on the demographic variables and risk factors associated with more severe disease.

\section{Descriptive Analysis of Asthmatics}

Our study not only highlights some of the previously published data regarding risk factors for severe COVID-19, but 
Table 4 Radiographic and Laboratory Findings in Adult Asthmatics Admitted for COVID-19

\begin{tabular}{|c|c|c|c|c|c|}
\hline & $\mathbf{N}$, if applicable ${ }^{a}$ & $\begin{array}{l}\text { Overall } \\
n=787\end{array}$ & $\begin{array}{c}\text { Male } \\
n=312\end{array}$ & $\begin{array}{c}\text { Female } \\
n=475\end{array}$ & p value \\
\hline \multicolumn{6}{|l|}{ Initial Chest Radiograph, n (\%) } \\
\hline Clear & & $93(11.8)$ & $31(9.9)$ & $62(13.1)$ & 0.23 \\
\hline Ground glass opacities (GGO) & & $95(12.1)$ & $44(14.1)$ & $51(10.7)$ & 0.19 \\
\hline Infiltrate/Opacity/Consolidation & & $580(73.7)$ & $229(73.4)$ & $351(73.9)$ & 0.94 \\
\hline Pleural effusion & & $16(2.0)$ & $6(1.9)$ & $10(2.1)$ & 1.00 \\
\hline \multirow{2}{*}{\multicolumn{6}{|c|}{$\begin{array}{l}\text { Most Severe Finding on Chest } \\
\text { Radiograph, } \mathbf{n}(\%)\end{array}$}} \\
\hline & & & & & \\
\hline Clear & & $56(7.1)$ & $21(6.7)$ & $35(7.4)$ & 0.84 \\
\hline Ground glass opacities (GGO) & & $134(17)$ & $59(18.9)$ & $75(15.8)$ & 0.30 \\
\hline Infiltrate/Opacity/Consolidation & & $604(76.7)$ & $238(76.3)$ & $366(77.1)$ & 0.87 \\
\hline Pleural effusion & & $34(4.3)$ & $13(4.2)$ & $21(4.4)$ & 1.00 \\
\hline Acute Kidney Injury, n (\%) & & $187(23.8)$ & $99(34.0)$ & $88(19.7)$ & $<0.001$ \\
\hline \multicolumn{6}{|l|}{ Labs, Mean (SD) } \\
\hline Initial ESR (mm/hr) & 322 & $62.7(33.6)$ & $58.6(33.6)$ & $65.6(33.4)$ & 0.06 \\
\hline Peak ESR (mm/hr) & 310 & $69.2(39.2)$ & $64.3(35.3)$ & $72.7(41.4)$ & 0.06 \\
\hline Initial WBC $(K / \mu L)$ & 785 & $8.44(7.04)$ & $8.67(5.21)$ & $8.28(8.02)$ & 0.41 \\
\hline Peak WBC $(K / \mu L)$ & 778 & $13.5(11.4)$ & $14.4(10.6)$ & $12.9(11.9)$ & 0.07 \\
\hline 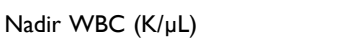 & 777 & $6.24(4.33)$ & $6.38(4.02)$ & $6.14(4.53)$ & 0.43 \\
\hline Initial lymphocyte count $(\mathrm{K} / \mu \mathrm{L})$ & 782 & $1.30(3.23)$ & $1.12(1.76)$ & $1.42(3.91)$ & 0.15 \\
\hline Lowest lymphocyte count $(\mathrm{K} / \mu \mathrm{L})$ & 768 & $0.963(1.79)$ & $0.89(1.84)$ & $1.0(1.76)$ & 0.37 \\
\hline Initial neutrophil count $(K / \mu L)$ & 782 & $7.27(10.3)$ & $7.37(7.5 \mathrm{I})$ & $7.21(11.7)$ & 0.81 \\
\hline Peak neutrophil count $(\mathrm{K} / \mu \mathrm{L})$ & 767 & $10.8(10.4)$ & $11.4(10.0)$ & $10.4(10.6)$ & 0.20 \\
\hline Initial procalcitonin (ng/mL) & 640 & $2.61(17.6)$ & $2.37(13.5)$ & $2.79(20.0)$ & 0.76 \\
\hline Initial AST (U/L) & 782 & $58.3(88.0)$ & $64.3(94.5)$ & $54.3(83.3)$ & 0.12 \\
\hline Peak AST (U/L) & 773 & $245(1360)$ & $264(1530)$ & $232(1230)$ & 0.74 \\
\hline Initial ALT (U/L) & 782 & $47.1(55.5)$ & $56.8(60.4)$ & $40.7(51.0)$ & $<0.001$ \\
\hline Peak ALT (U/L) & 773 & $175(777)$ & $212(66 I)$ & |5| (846) & 0.28 \\
\hline Tb cell CD3 absolute (cells $/ \mu \mathrm{L}$ ) & 77 & $551(364)$ & $48 \mid(257)$ & $630(447)$ & 0.07 \\
\hline Tb cell CD3\% & 78 & $74.9(114)$ & $61.5(12.1)$ & $89.8(165)$ & 0.27 \\
\hline Tb cell CD4 absolute (cells $/ \mu \mathrm{L}$ ) & 76 & $348(233)$ & $273(151)$ & $432(278)$ & 0.004 \\
\hline Tb cell CD4\% & 77 & $38.3(11.8)$ & $35.4(9.66)$ & $41.4(13.2)$ & 0.02 \\
\hline Tb cell CD8 absolute (cells $/ \mu \mathrm{L}$ ) & 76 & $203(165)$ & $198(161)$ & $208(|7|)$ & 0.78 \\
\hline Tb cell CD8\% & 77 & $24.6(18.0)$ & $28.2(22.3)$ & $20.7(10.6)$ & 0.06 \\
\hline Tb cell CD 9 absolute (cells $/ \mu \mathrm{L}$ ) & 41 & $164(123)$ & $136(81.9)$ & $187(145)$ & 0.18 \\
\hline Tb cell CDI656 absolute (cells/ $\mu \mathrm{L}$ ) & 41 & $487(2200)$ & $124(60.6)$ & 771 (2930) & 0.30 \\
\hline Tb cell CDI656\% & 41 & $16.4(9.69)$ & $16.4(9.85)$ & $16.3(9.80)$ & 0.96 \\
\hline Initial CRP absolute $(\mathrm{mg} / \mathrm{dL})$ & 699 & $16.3(29.7)$ & $16.6(28.3)$ & $16.0(30.7)$ & 0.79 \\
\hline Peak CRP absolute (mg/dL) & 691 & $22.5(40.8)$ & $23.2(36.1)$ & $22.1(43.7)$ & 0.73 \\
\hline Initial ferritin absolute $(\mathrm{ng} / \mathrm{mL})$ & 664 & $1020(1890)$ & $1480(2600)$ & $706(1070)$ & $<0.001$ \\
\hline Peak ferritin absolute $(\mathrm{ng} / \mathrm{mL})$ & 653 & $1730(4240)$ & $2610(5990)$ & $1120(2200)$ & $<0.001$ \\
\hline Initial D-dimer absolute $(\mathrm{ng} / \mathrm{mL})$ & 593 & $1470(4800)$ & $1680(5750)$ & $1320(4000)$ & 0.39 \\
\hline Peak D-dimer absolute $(\mathrm{ng} / \mathrm{mL})$ & 584 & $4010(9140)$ & $5140(10,700)$ & $3200(7720)$ & 0.02 \\
\hline Initial troponin absolute $(\mathrm{ng} / \mathrm{L})$ & 419 & $23.5(359)$ & $6.56(32.8)$ & $36.2(474)$ & 0.34 \\
\hline Peak troponin absolute $(\mathrm{ng} / \mathrm{L})$ & 381 & $51.4(586)$ & $34.9(236)$ & $64.1(752)$ & 0.59 \\
\hline Cytokine IL I absolute $(\mathrm{pg} / \mathrm{mL})$ & 37 & $14.1(38.6)$ & $5.59(2.26)$ & $22.1(53.2)$ & 0.19 \\
\hline Cytokine IL 6 absolute $(\mathrm{pg} / \mathrm{mL})$ & 61 & $132(427)$ & $96.6(268)$ & $173(562)$ & 0.52 \\
\hline Cytokine IL 10 absolute $(\mathrm{pg} / \mathrm{mL})$ & 41 & $17.3(20.5)$ & $18.2(18.9)$ & $16.3(22.7)$ & 0.76 \\
\hline Tb cell CDI9\% (cells/ $\mu \mathrm{L}$ ) & 42 & $20.6(16.7)$ & $17.7(9.45)$ & $22.8(20.5)$ & 0.30 \\
\hline
\end{tabular}

Note: ${ }^{a}$ Number of patients with respective lab values included in analyses.

Abbreviations: COVID-19, coronavirus disease 2019; SD, standard deviation; ESR, erythrocyte sedimentation rate; WBC, white blood cell count; AST, aspartate aminotransferase; ALT, alanine aminotransferase; Tb Cell, T and B cell subsets; CD, cluster of differentiation; CRP, c-reactive protein; IL, interleukin; mm/hr, millimeter per hour; $\mathrm{K} / \mu \mathrm{L}$, thousands per cubic milliliter; $\mathrm{ng} / \mathrm{mL}$, nanograms per milliliter; $\mathrm{U} / \mathrm{L}$, units per liter; cells/ $\mu \mathrm{L}$, cells per microliter; $\mathrm{mg} / \mathrm{dL}$, milligrams per deciliter; $\mathrm{ng} / \mathrm{L}$, nanograms per liter; pg/mL, picograms per milliliter. 
Table 5 Comorbidities in Adult Asthmatics Admitted for COVID-19

\begin{tabular}{|c|c|c|c|c|}
\hline & $\begin{array}{c}\text { Overall } \\
n=787\end{array}$ & $\begin{array}{c}\text { Male } \\
n=312\end{array}$ & $\begin{array}{c}\text { Female } \\
n=475\end{array}$ & p value \\
\hline Hypertension, n (\%) & $443(56.3)$ & |7| (54.8) & $272(57.3)$ & 0.55 \\
\hline Diabetes Mellitus, n (\%) & $256(32.5)$ & $85(27.2)$ & I7I (36.0) & 0.01 \\
\hline Cardiovascular, not Coronary Disease, n (\%) & $67(8.5)$ & $22(7.1)$ & $45(9.5)$ & 0.29 \\
\hline Coronary Artery Disease, n (\%) & $97(12.3)$ & $4 I(13.1)$ & $56(11.8)$ & 0.65 \\
\hline Obesity, n (\%) & $217(27.6)$ & $83(26.6)$ & $134(28.2)$ & 0.68 \\
\hline Cancer, n (\%) & $48(6.1)$ & $14(4.5)$ & $34(7.2)$ & 0.17 \\
\hline Immunosuppression, n (\%) & $26(3.3)$ & $10(3.2)$ & $16(3.4)$ & 1.00 \\
\hline Peripheral Vascular Disease, n (\%) & $20(2.5)$ & $5(1.6)$ & $15(3.2)$ & 0.26 \\
\hline Chronic Liver Disease, n (\%) & $8(1.0)$ & $4(1.3)$ & $4(0.8)$ & 0.81 \\
\hline Heart Failure, n (\%) & $53(6.7)$ & $18(5.8)$ & $35(7.4)$ & 0.47 \\
\hline Chronic Kidney Disease, n (\%) & $37(4.7)$ & $13(4.2)$ & $24(5.1)$ & 0.69 \\
\hline End-Stage Renal Disease, n (\%) & $25(3.2)$ & $9(2.9)$ & $16(3.4)$ & 0.86 \\
\hline
\end{tabular}

Abbreviation: COVID-19, coronavirus disease 2019.

Table 6 Clinical Outcomes in Adult Asthmatics by ICS Use

\begin{tabular}{|c|c|c|c|c|c|c|}
\hline & $\begin{array}{l}\text { Overall } \\
n=787\end{array}$ & $\begin{array}{c}\text { Any } \\
\text { ICS Use } \\
n=30 \text { I }\end{array}$ & $\begin{array}{c}\text { No ICS } \\
\text { Use } \\
n=486\end{array}$ & $\begin{array}{c}\text { Odds Ratio } \\
\text { (If Applicable) }\end{array}$ & $\begin{array}{c}\text { 95\% Confidence } \\
\text { Interval }\end{array}$ & p value \\
\hline Age (years), Mean (SD) & $59.8(16.4)$ & $63.5(15.2)$ & $57.6(16.7)$ & & & $<0.001$ \\
\hline Sex (female), $n$ (\%) & $475(60.4)$ & $193(64)$ & $282(58)$ & & & 0.10 \\
\hline Race, n (\%) & & & & & & 0.26 \\
\hline Asian & $65(8.3)$ & $26(8.6)$ & $39(8.0)$ & & & \\
\hline Black & $203(25.8)$ & 71 (23.6) & $132(27.2)$ & & & \\
\hline Other/Unknown ${ }^{\mathrm{a}}$ & $255(32.4)$ & $91(30.2)$ & $164(33.7)$ & & & \\
\hline White & $264(33.5)$ & $113(37.5)$ & $|5|(3||)$. & & & \\
\hline Ethnicity, n (\%) & & & & & & 0.07 \\
\hline Hispanic/Latino & $|7|(2 \mid .7)$ & $53(17.6)$ & $118(24.3)$ & & & \\
\hline Other & $43(5.5)$ & $15(5.0)$ & $28(5.8)$ & & & \\
\hline Non-Hispanic or Latino & $573(72.8)$ & $233(77.4)$ & $340(70.0)$ & & & \\
\hline Days on Ventilator, Mean (SD) ${ }^{b}$ & $13.9(15.2)$ & $15.0(16.1)$ & I3.| (14.6) & & $(-6.89,2.96)$ & 0.43 \\
\hline Length of Stay (days), Mean (SD) & $9.65(11.0)$ & $10.5(12.2)$ & $9.10(10.2)$ & & $(-3.08,10.5)$ & 0.09 \\
\hline Ventilator Support, n (\%) & $145(18.4)$ & $60(19.9)$ & $85(17.5)$ & 1.17 & $(0.81,1.70)$ & 0.39 \\
\hline Deaths, n (\%) & $144(18.3)$ & $61(20.3)$ & $83(17.1)$ & 1.15 & $(0.79,1.65)$ & 0.47 \\
\hline Discharged on Oxygen, n (\%) & $|3|(\mid 6.6)$ & $64(21.3)$ & $67(13.8)$ & $1.62^{\mathrm{c}}$ & $(1.10,2.38)$ & 0.01 \\
\hline ICU Admission, n (\%) & $194(24.7)$ & $81(26.9)$ & $113(23.3)$ & 1.22 & $(0.87,1.69)$ & 0.25 \\
\hline
\end{tabular}

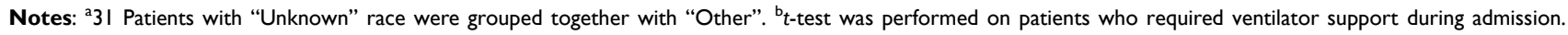
Patients without ventilator support were excluded from analysis. "Represents adjusted odds ratio for age.

Abbreviations: SD, standard deviation; ICS, inhaled corticosteroids; ICS + LABA, inhaled corticosteroids plus long-acting beta agonist; ICU, intensive care unit.

further elaborates on the role of asthma as a comorbid condition. While male gender is a well-documented risk factor for hospitalization with COVID-19, and males were $60.3 \%$ of the patients admitted to Northwell hospitals at the time of the cohort described in a previous study, ${ }^{3}$ our cohort demonstrated that among those admitted, there was a greater proportion of females, which is similar to another report on asthma and COVID-19. ${ }^{7}$ Race and ethnicity among our 
cohort were similar to the previous study. ${ }^{3}$ The BMI of asthmatics admitted with COVID-19 was elevated, reflecting the high co-prevalence of asthma and obesity. ${ }^{24}$ Interestingly, the obese-asthma phenotype, characterized by obesity, female sex, and non-eosinophilic inflammation, is one of the more severe asthma phenotypes, and based on our demographics, may have been over-represented in this hospitalized cohort. About $50 \%$ of admitted asthmatics were on a controller medication for asthma, whether ICS, ICS/ LABA or montelukast, indicating a more persistent phenotype among admitted asthmatics. Approximately $40 \%$ of the patients in our cohort used ICS on admission, which is less than another report of ICS use in asthmatics with COVID-19, although this study included both inpatients and outpatients. ${ }^{7}$ This contrasts with the $80 \%$ of the asthmatics reported to be using ICS in the UK prior to the pandemic. ${ }^{25}$ The overall rate of ICU admission, mechanical ventilation and mortality, as well as length of stay among asthmatics in our cohort was high, and reflective of the severe outcomes that were observed early in the pandemic. When comparing to the previous cohort, the rates of ICU admission and mechanical ventilation, as well as length of stay, were higher among asthmatics than the overall. While the mortality rate was similar, it is suspected that the mortality rate among the parent cohort is artificially elevated because they only evaluated disposition among those who were discharged or died at the time of manuscript submission. ${ }^{3}$

\section{Differences Based on Sex}

A higher percentage of women were on controllers than men, but this was not statistically significant. This may have been due to the demographic make-up of our cohort. There were differences in terms of race with a higher proportion of Black patients being female and a higher proportion of White patients being male. Underlying genetic differences may contribute to the variation in presentation based on race. A history of present or past tobacco use was higher in men, which may also play a role in the more severe outcomes in males ${ }^{26}$ and reflect differences in the general population. Women presented to the hospital less than a day earlier than men. Although this was not statistically significant, differences in perception of symptoms and when to seek medical attention may account for our findings. Women have worse asthma perception and more symptom experience than men. ${ }^{27}$ In terms of severity of COVID-19 endpoints, men had poorer outcomes in terms of need for mechanical ventilation; however, death, discharge on oxygen, ICU admission, days in ICU and LOS were not significantly different between sexes. Men were also more likely to receive tocilizumab, reflecting the greater severity of their disease, since this was a treatment reserved for the sickest patients. The vast majority of studies indicate that men are at higher risk for hospitalization, ICU admission, and death. ${ }^{28-30} \mathrm{It}$ is possible that while our data does support increased risk of severity given the higher proportion of men on mechanical ventilation and treated with tocilizumab, the number of subjects in our study did not allow the other severity endpoints to reach statistical significance. Alternatively, a possible interplay between female sex and asthma, may elevate the risk for death, discharge on oxygen, ICU admission, days in ICU and LOS among women, such that the sex differences in these severity outcomes is eliminated.

In general, the significant differences between men and women related to laboratory results reflected the clinical findings of more severe COVID-19 among men. For example, higher proportion of males had acute kidney injury than females, and males had higher ferritin, higher initial ALT, and peak D-dimer, which one would expect among individuals with more critical disease. In contrast, women had higher CD4 $\mathrm{T}$ cell counts. Whether this reflects a protective immunologic mechanism that contributed to less severe outcomes in women, is unknown, and should be further investigated.

\section{Laboratory Values and COVID-19 Endpoints}

Those subjects with higher levels of inflammatory markers on presentation were at risk for poorer COVID-19 outcomes, such as ICU admission, need for ventilation, and death. This is similar to findings of other cohorts of all patients, not specifically asthmatics. ${ }^{31-33}$ These markers of inflammation were noted to be lower among asthmatics compared to non-asthmatics. ${ }^{10}$

\section{Role of Inhaled Corticosteroids}

At the beginning of the pandemic, the use of corticosteroids to treat COVID-19 was fraught with controversy. Initially, there were concerns that corticosteroids could worsen the severity of COVID-19, but the RECOVERY trial demonstrated that carefully timed use of dexamethasone in hypoxic hospitalized patients significantly reduced mortality. ${ }^{15}$ 
Non-clinical studies have been encouraging regarding the use of steroids in SARS-CoV2. Among participants of the Severe Asthma Research Program, sputum mRNA expression of ACE2 and TMPRSS2 was downregulated among patients with ICS. ${ }^{22}$ However, in another study, pulmonary gene expression did not differ in patients with obstructive airway disease when they were on ICS. ${ }^{34}$ While our data did not demonstrate any increase or decrease in risk of mortality, ICU admission, need for mechanical ventilation or length of stay, asthmatics on ICS were 1.6 times more likely to be discharged on oxygen. This is the adjusted odds ratio when taking age into account, since those on ICS were significantly older than those not on ICS. Whether this reflects that the underlying severity of asthma or the use of ICS is associated with this outcome remains unknown at this time. Our finding that patients on ICS had significantly higher initial and peak CRP suggests that the association between ICS and discharge with oxygen is more likely due to the severity of their underlying disease. Since our study focused only on hospitalized patients, it is also unknown whether ICS increases the risk of hospitalization.

Specifically, among asthmatics, ICS could have both theoretical benefits and downsides in the setting of COVID-19. Type I interferons, which are critical for their antiviral activity, are impaired by high dose ICS. ${ }^{35}$ However, as discussed above, ICS may downregulate the receptors for viral entry and inhibit viral growth. Observational data regarding use of ICS in asthmatics with COVID is conflicting. A large observational cohort study in the UK demonstrated that COPD patients on ICS and asthmatics on higher dose ICS, did have a higher risk of mortality compared to those not on ICS, however the role of confounders such as disease severity could have played a role. ${ }^{36}$ The use of ICS seemed to protect asthmatics from hospitalization, in a study in Spain that analyzed the electronic health records of over 70,000 asthmatics. $^{37}$

Few studies have looked at ICS use in non-asthmatics in the management of COVID-19. Inhaled budesonide within 7 days of symptom onset resulted in reduction in COVID-19-related urgent care visits, ED visits or hospitalization, in addition to faster clinical recovery and less persistent symptoms. ${ }^{38}$ This was also demonstrated in a larger open-label study, but follow-up data has not yet been published. ${ }^{39}$ Several other clinical trials investigating the use of ICS are underway.
To our knowledge, this is the largest cohort of adult asthmatics hospitalized for COVID-19 early in the pandemic. The breadth and depth of this study is reflected in the large number of asthmatics, the diverse demographic background of the subjects, the range of asthma severity, as well as the large number of endpoints studied. Nevertheless, this study does have several limitations. The study would have been more informative if baseline demographics and COVID-19related endpoints were compared in asthmatics versus nonasthmatics. However, this was not possible due to our access to limited data. The retrospective nature of the study fails to capture all possible variables as a prospective study would be able to and is unable to control potential confounders. It is possible that all hospitalized asthmatics were not captured in our cohort. We were not able to assess compliance with ICS or able to clinically determine the asthma severity and level of asthma control for each patient. In addition, this study evaluated asthma patients hospitalized early in the pandemic. Many asthmatics with COVID-19 are likely managed in the outpatient setting. A prospective study which follows asthmatics from time of diagnosis to time of resolution of symptoms would be ideal in assessing the true clinical course of all asthmatics, not just those that were hospitalized. Furthermore, with the advent of new therapeutics, widespread immunization in the local area and greater knowledge regarding the optimal management of COVID-19, the clinical outcomes of patients have surely changed. While our findings did not support an increased risk of mortality among asthmatics, the risk of morbidity in asthmatics hospitalized for COVID-19, was increased compared to the previous study. Conflicting findings may be reflective of the different asthma phenotypes and that some phenotypes are more susceptible to need for hospitalization or worse COVID-19 outcomes. Ultimately, a prospective cohort following asthmatics from time of diagnosis in the outpatient and inpatient settings is essential. Fortunately, in areas with high vaccination rates, the risk of severe disease is decreasing. However, large pockets of the globe have yet to be vaccinated, and the emergence of SARS-CoV2 variants, which may not be completely susceptible to vaccine immunity, is a valid concern. Furthermore, it remains to be determined if immunity through vaccination confers the same type of protection for asthmatics compared to others, and for asthmatics on ICS compared to those not on ICS. As the virus mutates, the role of how medications and other comorbidities modify risk of COVID-19 severity is an issue that researchers must still investigate. 


\section{Conclusion}

Outcomes of asthmatics hospitalized with COVID-19 were associated with sex and ethnicity. ICS use was associated with discharge with oxygen and higher CRP levels. The pandemic's fluid dynamics require prospective trials of asthmatics.

\section{Abbreviations}

COVID-19, coronavirus disease 2019; ICS, inhaled corticosteroids; ICU, intensive care unit; SARS-CoV2, severe acute respiratory syndrome coronavirus 2; GINA, Global Initiative for Asthma; ACE2, angiotensin converting enzyme 2; TMSP2, transmembrane serine protease 2; EMR, electronic medical record; BMI, body mass index; LOS, length of stay; CXR, chest X-ray; WBC, white blood cell count; ALC, absolute lymphocyte count; ANC, absolute neutrophil count; CRP, C-reactive protein; ESR, erythrocyte sedimentation rate; AST, aspartate aminotransferase; ALT, alanine aminotransferase.

\section{Acknowledgments}

We would like to acknowledge the following individuals for their assistance with data collection: Tarif Anzum, MD; Alexander Babazadeh, MD; Gina Coscia MD, Sanjiv Godse, MD; Stefanie Griffin, MD; Jonathan Ilowite, MD; Irina Katayeva, MD; Alissa McInerney, MD; George Mundanchira, MD; Annabelle Quizon, MD; Steven Saunders, MD. We would like to acknowledge Jane Cerise, PhD, for her help with statistics and Artemio Jongco, MD, PhD, MPH, for his review of statistics and of the overall manuscript. We would like to acknowledge the Northwell Health COVID-19 Research Consortium for their contribution to data procurement.

\section{Disclosure}

The authors report no conflicts of interest in this work.

\section{References}

1. CDC. People with certain medical conditions. Available from: https:// www.cdc.gov/coronavirus/2019-ncov/need-extra-precautions/peoplewith-medical-conditions.html. Accessed August 19, 2021.

2. Li Q, Guan X, Wu P, et al. Early transmission dynamics in Wuhan, China, of novel coronavirus-infected pneumonia. $N$ Engl $J$ Med. 2020;382(13):1199-1207. doi:10.1056/NEJMoa2001316

3. Richardson S, Hirsch JS, Narasimhan M, et al. Presenting characteristics, comorbidities, and outcomes among 5700 patients hospitalized with COVID-19 in the New York City Area. JAMA. 2020;323 (20):2052-2059. doi:10.1001/jama.2020.6775
4. Docherty AB, Harrison EM, Green CA, et al. Features of 16,749 hospitalised UK patients with COVID-19 using the ISARIC WHO clinical characterisation protocol. BMJ. 2020;369:m1985. doi:10.1136/bmj.m1985

5. Mendes NF, Jara CP, Mansour E, Araujo E, Velloso LA. Asthma and COVID-19 -A systematic review. Allergy Asthma Clin Immunol. 2021;17(1). doi:10.22541/au.159118771.11841404

6. Li X, Xu S, Yu M, et al. Risk factors for severity and mortality in adult COVID-19 inpatients in Wuhan [published online ahead of print April 12, 2020]. J Allergy Clin Immunol. 2020;146:110-118. doi:10.1016/j.jaci.2020.04.006

7. Chhiba KD, Patel GB, Vu THT, et al. Prevalence and characterization of asthma in hospitalized and nonhospitalized patients with COVID-19. J Allergy Clin Immunol. 2020;146(2):307-314.e4. PMID: 32554082; PMCID: PMC7295471. doi: 10.1016/j. jaci.2020.06.010

8. Singer AJ, Morley EJ, Meyers K, et al. Cohort of 4404 persons under investigation for COVID-19 in a NY hospital and predictors of ICU care and ventilation [published online ahead of print April 23, 2020]. Ann Emerg Med. 2020. doi:10.2139/ssrn.3583678

9. Lieberman-Cribbin W, Rapp J, Alpert N, Tuminello S, Taioli E. The impact of asthma on mortality in patients with COVID-19. Chest. 2020;158(6):2290-2291. doi:10.1016/j.chest.2020.05.575

10. Lovinsky-Desir S, Deshpande DR, De A, et al. Asthma among hospitalized patients with COVID-19 and related outcomes. $J$ Allergy Clin Immunol. 2020;146(5):1027-1034.e4. PMID: 32771560; PMCID: PMC7409831. doi: 10.1016/j.jaci.2020.07.026

11. Williamson EJ, Walker AJ, Bhaskaran K, et al. Factors associated with COVID-19-related death using OpenSAFELY. Nature. 2020;584 (7821):430-436. doi:10.1038/s41586-020-2521-4

12. Lee SC, Son KJ, Han CH, Jung JY, Park SC. Impact of comorbid asthma on severity of coronavirus disease (COVID-19). Sci Rep. 2020;10(1):21805. PMID: 33311519; PMCID: PMC7733453. doi:10.1038/s41598-020-77791-8

13. Caminati M, Vultaggio A, Matucci A, et al. Asthma in a large COVID-19 cohort: prevalence, features, and determinants of COVID-19 disease severity. Respir Med. 2021;176:106261. PMID: 33260107; PMCID: PMC7688414. doi:10.1016/j.rmed.2020.106261

14. Atkins JL, Masoli JAH, Delgado J, et al. Preexisting comorbidities predicting COVID-19 and mortality in the UK biobank community cohort. J Gerontol a Biol Sci Med Sci. 2020;75(11):2224-2230. PMID: 32687551; PMCID: PMC7454409. doi:10.1093/gerona/glaa183

15. Horby P, Lim WS, Emberson JR, et al.; RECOVERY Collaborative Group. Dexamethasone in hospitalized patients with Covid-19. $N$ Engl J Med. 2021; 384(8):693-704. PMID: 32678530; PMCID: PMC7383595. doi:10.1056/NEJMoa2021436

16. Sterne JAC, Murthy S, Diaz JV, et al.; WHO Rapid Evidence Appraisal for COVID-19 Therapies (REACT) Working Group. Association between administration of systemic corticosteroids and mortality among critically ill patients with COVID-19: a meta-analysis. JAMA. 2020;324(13):1330-1341. PMID: 32876694; PMCID: PMC7489434. doi:10.1001/jama.2020.17023

17. Choi JC, Jung SY, Yoon UA, et al. Inhaled Corticosteroids and COVID-19 risk and mortality: a nationwide cohort study. J Clin Med. 2020;9(11):3406. PMID: 33114246; PMCID: PMC7690894. doi:10.3390/jcm9113406

18. Matsuyama S, Kawase M, Nao N, et al. The inhaled steroid ciclesonide blocks SARS-CoV-2 RNA replication by targeting the viral replication-transcription complex in cultured cells. $J$ Virol. 2020;95 (1):e01648-20. PMID: 33055254; PMCID: PMC7737752. doi:10.1128/JVI.01648-20

19. Jeon S, Ko M, Lee J, et al. Identification of antiviral drug candidates against SARS-CoV-2 from FDA-approved drugs. Antimicrob Agents Chemother. 2020;64(7):e00819-20. PMID: 32366720; PMCID: PMC7318052. doi:10.1128/AAC.00819-20 
20. Hoffmann M, Kleine-Weber H, Schroeder S, et al. SARS-CoV-2 cell entry depends on ACE2 and TMPRSS2 and is blocked by a clinically proven protease inhibitor. Cell. 2020;181(2):271-280.e8. PMID: 32142651; PMCID: PMC7102627. doi: 10.1016/j.cell.2020.02.052

21. Jackson DJ, Busse WW, Bacharier LB, et al. Association of respiratory allergy, asthma, and expression of the SARS-CoV-2 receptor ACE2. J Allergy Clin Immunol. 2020;146(1):203-206.e3. PMID: 32333915; PMCID: PMC7175851. doi: 10.1016/j.jaci.2020.04.009

22. Kimura H, Francisco D, Conway M, et al. Type 2 inflammation modulates ACE2 and TMPRSS2 in airway epithelial cells. J Allergy Clin Immunol. 2020;146(1):80-88.e8. PMID: 32422146; PMCID: PMC7227558. doi: 10.1016/j.jaci.2020.05.004

23. Peters MC, Sajuthi S, Deford P, et al. COVID-19-related genes in sputum cells in asthma. relationship to demographic features and corticosteroids. Am J Respir Crit Care Med. 2020;202(1):83-90. Erratum in: Am $J$ Respir Crit Care Med. 2020 Dec 15;202(12):1744-1746. PMID: 32348692; PMCID: PMC7328313. doi:10.1164/rccm.202003-0821OC

24. Miethe S, Karsonova A, Karaulov A, Renz H. Obesity and asthma. J Allergy Clin Immunol. 2020;146(4):685-693. PMID: 33032723. doi:10.1016/j.jaci.2020.08.011

25. Bloom CI, Saglani S, Feary J, Jarvis D, Quint JK. Changing prevalence of current asthma and inhaled corticosteroid treatment in the UK: population-based cohort 2006-2016. Eur Respir J. 2019;53 (4):1802130. PMID: 30765507. doi:10.1183/13993003.02130-2018

26. Zhao Q, Meng M, Kumar R, et al. The impact of COPD and smoking history on the severity of COVID-19: a systemic review and meta-analysis. $J$ Med Virol. 2020;92(10):1915-1921. PMID: 32293753; PMCID: PMC7262275. doi: 10.1002/jmv.25889

27. Colombo D, Zagni E, Ferri F, Canonica GW; PROXIMA study centers. Gender differences in asthma perception and its impact on quality of life: a post hoc analysis of the PROXIMA (Patient reported outcomes and Xolair $^{\circledR}$ in the management of asthma) study. Allergy Asthma Clin Immunol. 2019;15(1):65. PMID: 31708980; PMCID: PMC6833238. doi:10.1186/s13223-019-0380-Z

28. Li J, Huang DQ, Zou B, et al. Epidemiology of COVID-19: a systematic review and meta-analysis of clinical characteristics, risk factors, and outcomes. J Med Virol. 2021;93(3):1449-1458. PMID: 32790106; PMCID: PMC7436673. doi: 10.1002/jmv.26424

29. Gebhard C, Regitz-Zagrosek V, Neuhauser HK, Morgan R, Klein SL. Impact of sex and gender on COVID-19 outcomes in Europe. Biol Sex Differ. 2020;11(1):29. PMID: 32450906; PMCID: PMC7247289. doi:10.1186/s13293-020-00304-9

30. Jin JM, Bai P, He W, et al. Gender differences in patients with COVID-19: focus on severity and mortality. Front Public Health. 2020;8:152. PMID: 32411652; PMCID: PMC7201103. doi:10.3389/ fpubh.2020.00152
31. Zhang L, Yan X, Fan Q, et al. D-dimer levels on admission to predict in-hospital mortality in patients with Covid-19. J Thromb Haemost. 2020;18(6):1324-1329. PMID: 32306492; PMCID: PMC7264730. doi: $10.1111 /$ jth. 14859

32. Shang W, Dong J, Ren Y, et al. The value of clinical parameters in predicting the severity of COVID-19. J Med Virol. 2020;92 (10):2188-2192. PMID: 32436996; PMCID: PMC7280691. doi: $10.1002 / \mathrm{jmv} .26031$

33. Henry BM, de Oliveira MHS, Benoit S, Plebani M, Lippi G. Hematologic, biochemical and immune biomarker abnormalities associated with severe illness and mortality in coronavirus disease 2019 (COVID-19): a meta-analysis. Clin Chem Lab Med. 2020;58 (7):1021-1028. PMID: 32286245. doi:10.1515/cclm-2020-0369

34. Maes T, Bracke K, Brusselle GG. COVID-19, asthma, and inhaled corticosteroids: another beneficial effect of inhaled corticosteroids? Am J Respir Crit Care Med. 2020;202(1):8-10. PMID: 32437628; PMCID: PMC7328335. doi:10.1164/rccm.202005-1651ED

35. Simpson JL, Carroll M, Yang IA, et al. Reduced antiviral interferon production in poorly controlled asthma is associated with neutrophilic inflammation and high-dose inhaled corticosteroids. Chest. 2016;149 (3):704-713. PMID: 26836898. doi: 10.1016/j.chest.2015.12.018

36. Schultze A, Walker AJ, MacKenna B, et al.; OpenSAFELY Collaborative. Risk of COVID-19-related death among patients with chronic obstructive pulmonary disease or asthma prescribed inhaled corticosteroids: an observational cohort study using the OpenSAFELY platform. Lancet Respir Med. 2020; 9 (11):1106-1120. PMID: 32979987; PMCID: PMC7515601. doi:10.1016/S2213-2600(20)30415-X

37. Izquierdo JL, Almonacid C, González $\mathrm{Y}$, et al. The impact of COVID-19 on patients with asthma. Eur Respir J. 2021;57 (3):2003142. PMID: 33154029; PMCID: PMC7651839. doi: $10.1183 / 13993003.03142-2020$

38. Ramakrishnan S, Nicolau DV Jr, Langford B, et al. Inhaled budesonide in the treatment of early COVID-19 (STOIC): a Phase 2, open-label, randomised controlled trial. Lancet Respir Med. 2021;9 (7):763-772; Erratum in: Lancet Respir Med. 2021 Jun;9(6):e55. PMID: 33844996; PMCID: PMC8040526. doi: 10.1016/S22132600(21)00160-0

39. Yu LM, Bafadhel M, Dorward J, et al.; PRINCIPLE Trial Collaborative Group. Inhaled budesonide for COVID-19 in people at high risk of complications in the community in the UK (PRINCIPLE): a randomised, controlled, open-label, adaptive platform trial. Lancet. 2021:S0140-S6736. PMID: 34388395; PMCID: PMC8354567. doi:10.1016/S0140-6736(21)01744-X

\section{Publish your work in this journal}

The Journal of Asthma and Allergy is an international, peer-reviewed open-access journal publishing original research, reports, editorials and commentaries on the following topics: Asthma; Pulmonary physiology; Asthma related clinical health; Clinical immunology and the immunological basis of disease; Pharmacological interventions and new therapies. The manuscript management system is completely online and includes a very quick and fair peer-review system, which is all easy to use. Visit http://www.dovepress.com/testimonials.php to read real quotes from published authors. 